\title{
The effect of hyperlipidemia on bone graft regeneration of peri-implantal created defects in rabbits
}

\author{
Mehmet Bugrul Tekin ${ }^{1}$ and Hulya Toker $^{2^{*}}$ (D)
}

\begin{abstract}
Aim: It is reported that hyperlipidemia affects quality and density of bone and adversely affects wound healing. The effect of hyperlipidemia on implant osseointegration and peri-implant defect regeneration has not been fully explained. The purpose of this study was to examine the effects of hyperlipidemia on the healing potential of the materials used for peri-implant bone regeneration and implant stability.

Materials and methods: Twelve male, New Zealand rabbits were used in this study. Half of the rabbits were fed a $2 \%$ cholesterol diet for 8 weeks to induce hypercholesterolemia. Peri-implant defects $(7 \mathrm{~mm}$ diameter) were created in the tibias of rabbits and placed implants $(3.3 \mathrm{~mm}$ in diameter). This study was conducted as a split-mouth design. Animals were randomly divided into two groups: (1) hypercholesterol+autogenous graft group and hypercholesterol+xenograft group $(n=6)$, and (2) autogenous graft and xenograft groups as controls $(n=6)$. At 8 weeks after surgery, the rabbits were euthanized. During implant surgery and at 8 weeks, implant stability was measured with resonance frequency analysis (RFA values). Bone-to-implant contact (BIC) was analyzed via histomorphometric analysis.
\end{abstract}

Results: Hyperlipidemic groups showed significantly lower BIC values than those of the control groups at 8 weeks $(p<0.05)$. According to baseline RFA readings, there was no significant difference between control and hyperlipidemic groups ( $p>0.05$ ). The hypercholesterol+autogenous graft group had significantly lower RFA readings and BIC values than the hypercholesterol+xenograft group at 8 weeks $(p<0.05)$.

Conclusion: Within the limitations of this study, it was found that hyperlipidemia may negatively affect the implant stability especially in the autogenous group and also, may decrease peri-implant bone regeneration. However, further studies are necessary to confirm these results more.

Keywords: Peri-implant, Hyperlipidemia, Bone regeneration, Implant stability

\section{Summary}

These findings suggest that hyperlipidemia reduced bone graft regeneration and decreased implant stability in experimental peri-implant defects in rabbits.

Dental implant survival is mainly dependent on successful osseointegration following placement. Any alteration of this biological process may adversely affect the success rate. Also, the long-term prognosis is adversely affected by inadequate bone volume at implant sites. There

\footnotetext{
* Correspondence: tokerhulya@gmail.com

${ }^{2}$ Periodontology Department, Gulhane Faculty of Dentistry, University of Health Sciences, Ankara, Turkey

Full list of author information is available at the end of the article
}

are several risk factors that were defined as implant failure. One of the risks of implant's failure depends on the systemic health of the subject (such as diabetes mellitus, osteoporosis, smoking) Recently, some authors suggested that there is a relationship between hypercholesterolemia and dental implant osseointegration [1,2].

Hyperlipidemia is a state with an abnormal lipid profile, which is characterized by elevated blood concentrations of triglycerides, elevated levels of total cholesterol and LDL, and decreased levels of HDL cholesterol [3]. Hyperlipidemia is associated with several diseases such as atherosclerosis and osteoporosis. The National Health and Nutrition Examination Survey (NHANES III) reported 
that $63 \%$ of osteoporotic patients have hyperlipidemia [4]. Furthermore, in the USA, among the approximately 300,000 implants placed due to fractures every year, 5-10\% exhibit impaired healing or osseointegration [5, 6]. The main mechanisms of the relationship between hyperlipidemia and bone tissue metabolism are the involved aspects of some metabolic changes, including lower bone mineral density, increase in the number of osteoclasts, and the inhibition of osteoblastic activity. However, several investigators suggested that lipidlowering drugs, such as statins, had beneficial effects on bone metabolism and also favorable effects on statins observed on osteogenesis around implants [7].

Bone augmentation procedures stimulate or facilitate the growth of new bone into the defected site. Although autologous or autogenous bone grafts are the ideal graft material for use in periodontal and implant surgeries, its use is restricted by the limitation of graft volume available, donor site morbidity, and prolongation of the operation. Thus, various alternative materials have been used including allografts and xenografts [8-10]. Xenografts are bone grafts that are derived from a donor of different species and are accepted as an osteoconductive material [11]. It was reported that xenograft subsequently replaced newly formed bone and bone apposition around titanium implants [12]. Furthermore, Hockers et al. found that deproteinized bone mineral and autogenic bone grafts appeared to be equally well integrated into the regenerating bone around implants [13].

To the best of our knowledge, peri-implant bone graft healing in the presence of hyperlipidemia has not been studied extensively yet. However, there is only one study that has investigated the effects of hyperlipidemia on implant osseointegration in mice via implant push-in test and micro-CT analysis. In that study, Keuroghlian [1] found that high-fat diet-fed mice had significantly increased implant loss as well as decreased formation and strength of the bone-to-implant interface. Therefore, based on these unfavorable aspects of hyperlipidemia, the two hypotheses that will be tested is that high levels of cholesterol can impair bone graft regeneration around a peri-implantal defect and can decrease implant stability. Therefore, the goal of this study was to investigate the histomorphometric evaluation of xenograft and autogenous bone graft healing in high-fat diet-induced animals with peri-implant bone defects.

\section{Materials and methods}

\section{Animals}

Prior to the study, the protocol was approved by the Animal Ethics Committee of Cumhuriyet University Faculty of Medicine under protocol number 65202830/130. Twelve New Zealand male rabbits (6-months old), with a mean weight of $3-3.2 \mathrm{~kg}$, were included in this study.
The animals were maintained in individual cages at $21^{\circ}$ $\mathrm{C}$ with 12-h day/night cycles and free to access to food and fresh water.

\section{Induction of hyperlipidemia}

Hyperlipidemia was induced on half of the animals by a 2\% high-lipid diet including 95\% pure cholesterol extract (Acros Organics, Geel, Belgium). Animals started on the diet 8 weeks prior to implant placement. Hyperlipidemic diet increased the total cholesterol levels fivefold at 8 weeks (Table 1), then experimental protocols were conducted. Also, blood samples were collected for the analyses of triglyceride, total cholesterol [14], and fraction (HDL and LDL) at the beginning, 4 weeks, and 8 weeks during the experiment.

This study was conducted as a split-mouth design. After the hyperlipidemia induction, two implants were placed in every animal and the peri-implant defect that was restored had different treatment protocols, and the study groups were the following:

- Hypercholesterol+autogenous graft (HPL+AG) group and hypercholesterol+xenograft $(\mathrm{HPL}+\mathrm{XG})$ group $(n=6)$,

- Autogenous graft (AG) and xenograft (XG) groups as controls $(n=6)$.

\section{Surgical procedures}

All animals were anesthetized with an intramuscular injection of ketamine $(40 \mathrm{mg} / \mathrm{kg}$, Eczacibasi Ilac Sanayi, Istanbul, Turkey) and xylazine $(10 \mathrm{mg} / \mathrm{kg}$, Bioveta a.s., Komenskeho, Chezh Republic). The surgical site was disinfected with iodine solution and shaved. A 2-cm incision was made for rising full-thickness flap in the tibia. Circumferential defect (7-mm wide and 4-mm depth)

Table 1 Biochemical parameters of study groups during hyperlipidemia induction prior to implant placement protocol $($ mean \pm SD)

\begin{tabular}{llllll}
\hline & & Total cholesterol & Triglyceride & HDL & LDL \\
\hline AG & Baseline & $28 \pm 1.1$ & $22 \pm 1.5$ & $20 \pm 2$ & $14 \pm 1.5$ \\
& 4 weeks & $27 \pm 1.6$ & $21 \pm 1.6$ & $19 \pm 1.4$ & $15 \pm 2$ \\
& 8 weeks & $31 \pm 1.9$ & $25 \pm 2$ & $20 \pm 1.4$ & $18 \pm 1.9$ \\
XG & Baseline & $25 \pm 1.4$ & $20 \pm 1.6$ & $18 \pm 1.9$ & $17 \pm 1.5$ \\
& 4 weeks & $30 \pm 1.5$ & $24 \pm 1.5$ & $18 \pm 1.5$ & $20 \pm 1.6$ \\
& 8 weeks & $29 \pm 1.8$ & $19 \pm 1.1$ & $20 \pm 1.4$ & $21 \pm 1.4$ \\
HPL+AG & Baseline & $25 \pm 2.5$ & $23 \pm 2.2$ & $18 \pm 1.2$ & $15 \pm 1.3$ \\
& 4 weeks & $61 \pm 2.1$ & $55 \pm 1.5$ & $38 \pm 1.7$ & $34 \pm 2.6$ \\
& 8 weeks & $101 \pm 2$ & $90 \pm 1.5$ & $39 \pm 1.5$ & $38 \pm 2.3$ \\
HPL+XG & Baseline & $22 \pm 1.8$ & $18 \pm 2$ & $17 \pm 2$ & $16 \pm 1.9$ \\
& 4 weeks & $70 \pm 2.2$ & $53 \pm 1.3$ & $36 \pm 2.1$ & $37 \pm 2$ \\
& 8 weeks & $110 \pm 1.6$ & $94 \pm 1.4$ & $38 \pm 1.6$ & $41 \pm 1.5$ \\
\hline
\end{tabular}


was created using trephine bur (MIS Implant Technologies, Sholomi, Israel) with a low-speed handpiece under continuous irrigation with sterile saline at both sides of the tibia. Implant osteotomy was performed at $800 \mathrm{rpm}$ and the submerged type implants $(\varnothing 3.3 \mathrm{~mm} \times 8 \mathrm{~mm}, \mathrm{RBM}$ surface, İmplance, Trabzon, Turkey) were placed. Circumferential defects were grafted with either AG or XG. Bovine bone (Bio-Oss, Geistlich Pharma North America, NJ, USA) with a form of granules measuring $0.25-1 \mathrm{~mm}$ in size was used in this study. Furthermore, the bone obtained during the defect formation was crushed in the bone crusher (Schwert, Seitingen/Oberflacht, Germany) and then implanted into defects as an autogenous graft.

The wound was sutured and postoperative antibiotic (ceftriaxone, intramuscularly (i.m.) $30 \mathrm{mg} / \mathrm{kg}, 3$ days $1 \times 1$ ) and analgesic (carprofen, i.m. $4 \mathrm{mg} / \mathrm{kg}, 3$ days, $1 \times 1$ ) were administered to animals to prevent postoperative infection and pain.

The animals were euthanized (by an overdose of 3\% pentobarbital sodium) at 8 weeks after implant placement.

\section{Resonance frequency analysis measurement}

The RFA was measured soon after insertion of the implant (baseline) and after 8 weeks before removal of bone blocks from tibiae, using a resonance frequency analysis device (Osstell Mentor; Integration Diagnostics AB, Göteborg, Sweden). A Smartpeg (Integration Diagnostics $A B$ ) was screwed into each implant and tightened to approximately $5 \mathrm{~N}-\mathrm{cm}$. The transducer probe was aimed at the small magnet at the top of the Smartpeg at a distance of 2 or $3 \mathrm{~mm}$ and held stable during the pulsing until the instrument beeped and displayed the RFA value. The measurements were taken twice in the buccolingual direction and twice in the mesiodistal direction. The mean of the four measurements was recorded as the RFA values.

\section{Histomorphometric analysis}

Bone block sections including implants were removed and the samples were fixed in $4 \%$ neutral buffered formalin for $24 \mathrm{~h}$ then consecutively dehydrated using alcohol and embedded with resin (Tecnovit 7200 VLC, Heraus Kulzer GmbH, Wehrheim, Germany). The resin blocks were polymerized and sectioned in a mesiodistal plane using a cutting-grinding unit (Exakt $300 \mathrm{CL}$, Apparatbau, Norderstedt, Hamburg, Germany) in 300- $\mu$ m thickness. After, the thickness of sections was carefully reduced to $40 \mu \mathrm{m}$ using a micro-grinding system (Exakt $400 \mathrm{CS}$, Apparatbau, Norderstedt, Hamburg, Germany). Following this, the samples were stained with toluidine blue.

The histomorphometric analysis was performed by a single examiner (BT) who was masked from the samples' identities. The images of the sections in all groups were captured by a digital camera connected to a light microscope (Olympus ${ }^{\bullet}$ CX41, Tokyo, Japan) and loaded to a computer. The image analysis software (Analysis LS Research, Version 5.0, Olympus Soft Imaging Solutions) was used for the measurements. Bone-to-implant contact (BIC) was measured as the amount of direct contact between the bone and the implant surface. Also, the amount of BIC was recorded as micrometers.

\section{Statistical analysis}

Statistical analyses were performed with SPSS, version 22 (IBM Corporation, New York, USA). KolmogorovSmirnov test was performed to test the normality of the data distribution. Mann-Whitney $U$ test was used to compare groups. Wilcoxon test was performed in the intragroup analysis. The data were presented as a mean \pm standard deviation and $p<0.05$ was considered statistically significant.

\section{Results}

The animals tolerated the surgical treatment during the experiment well. However, one animal from the hyperlipidemic group and one animal from nonhyperlipidemic group died from an infection. Also, at the end of the study, the clinical examination performed prior to sacrifice disclosed that all implants were stable and that the surrounding mucosae were clinically noninflamed.

The means of the biochemical analyses were presented in Table 2. During the study period, no significant differences were found in lipid biomarkers (TG, LDL, HDL, TC) and these levels were also higher in hyperlipidemic groups than those of the non-hyperlipidemic group $(p<0.05)$.

In Fig. 1 were shown the mean value of BIC of all groups. After 8 weeks, BIC value was greater in the control groups compared to their respective hyperlipidemic

Table 2 Biochemical parameters of study groups during experiment (mean $\pm \mathrm{SD}$ )

\begin{tabular}{llllll}
\hline & & Total cholesterol & Triglyceride & HDL & LDL \\
\hline AG & Baseline & $28 \pm 2.4$ & $22 \pm 1.5$ & $20 \pm 2$ & $14 \pm 1.4$ \\
& 4 weeks & $27 \pm 1.1$ & $21 \pm 2$ & $19 \pm 1.2$ & $15 \pm 2$ \\
& 8 weeks & $31 \pm 1.9$ & $25 \pm 1.6$ & $20 \pm 1.5$ & $18 \pm 1.8$ \\
XG & Baseline & $25 \pm 1.5$ & $20 \pm 1.5$ & $18 \pm 1.9$ & $17 \pm 1.3$ \\
& 4 weeks & $30 \pm 1.6$ & $24 \pm 1.7$ & $18 \pm 1.4$ & $20 \pm 1.4$ \\
& 8 weeks & $29 \pm 1.5$ & $19 \pm 2.1$ & $20 \pm 1.5$ & $21 \pm 1.7$ \\
HPL+AG & Baseline & $101 \pm 2.5$ & $90 \pm 2.2$ & $39 \pm 1.5$ & $38 \pm 1.3$ \\
& 4 weeks & $105 \pm 1.5$ & $98 \pm 1.2$ & $40 \pm 1.4$ & $41 \pm 2.7$ \\
& 8 weeks & $110 \pm 1.4$ & $100 \pm 1.8$ & $41 \pm 1.7$ & $54 \pm 1.7$ \\
HPL+XG & Baseline & $110 \pm 2$ & $94 \pm 3.1$ & $38 \pm 2.1$ & $41 \pm 1.9$ \\
& 4 weeks & $113 \pm 2.2$ & $96 \pm 2.2$ & $40 \pm 2$ & $43 \pm 2$ \\
& 8 weeks & $114 \pm 1.5$ & $101 \pm 2.1$ & $39 \pm 1.4$ & $47 \pm 1.6$ \\
\hline
\end{tabular}




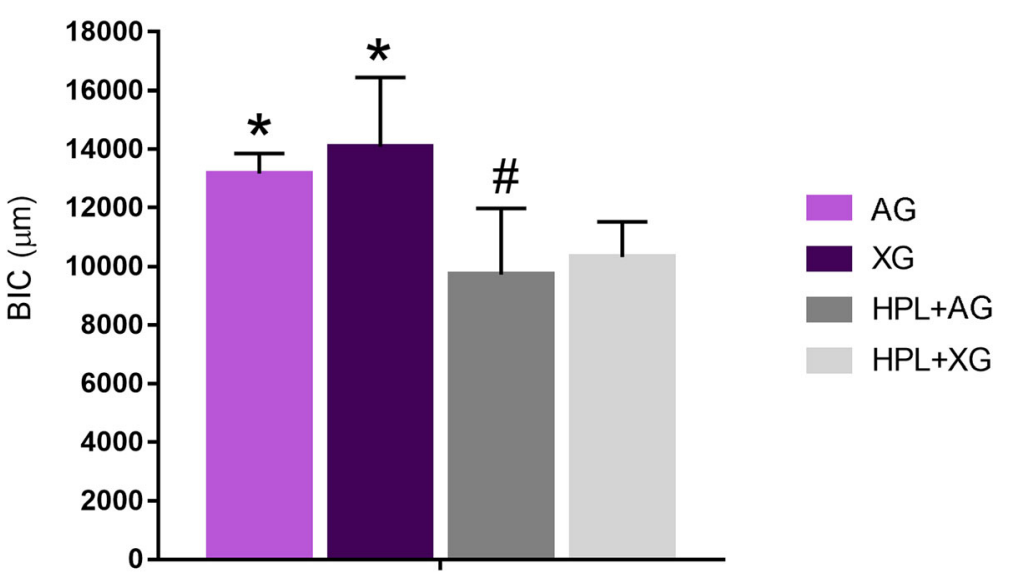

Fig. 1 Mean BIC value of study groups at 8 weeks. ${ }^{*} p<0.05$ versus HPL+AG and HPL+XG group, ${ }^{\#} p<0.05$ versus HPL+XG group ( $n$ of each group is 5 )

groups as shown in Fig. $2(p<0.05)$. Also, there was a significantly higher $\mathrm{BIC}$ value in the $\mathrm{HPL}+\mathrm{XG}$ group than those in the HPL+AG group $(p<0.05)$. However, there was no significant difference in BIC value between the AG and XG groups $(p>0.05)$.

RFA was measured at the time of implant placement and after 8 weeks (Fig. 3). According to intragroup comparisons, there was a significant difference in RFA readings between baseline and 8 weeks $(p<0.05)$. In all

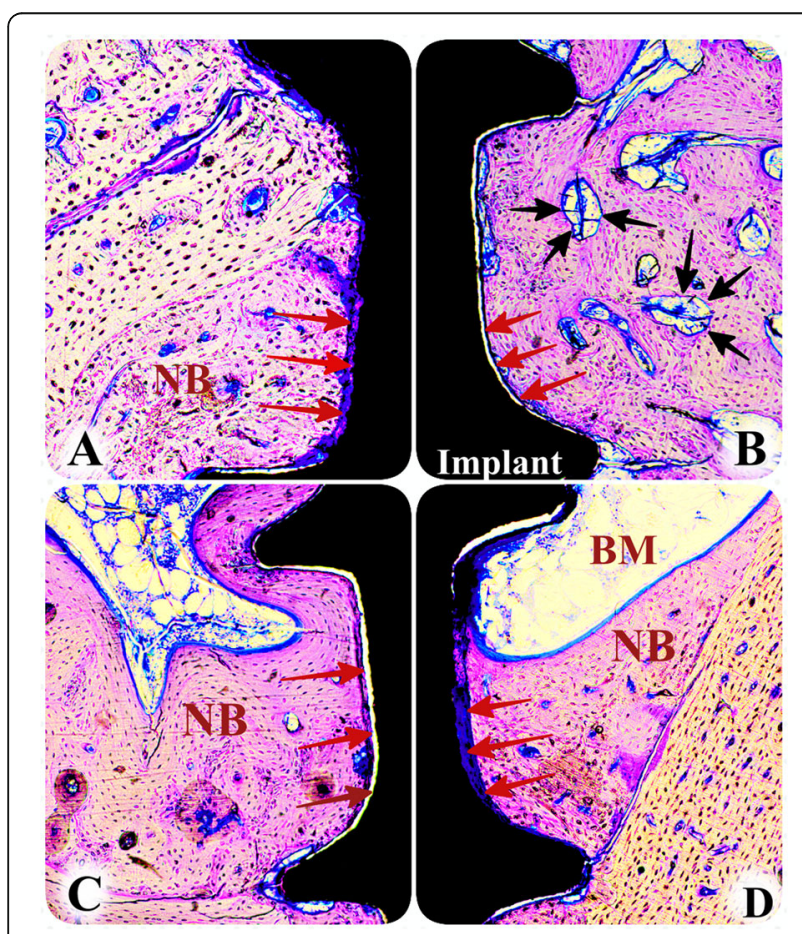

Fig. 2 Histologic views of the study groups at 8 weeks (toluidine blue, $\times 10$ ). a AG group, b XG group, $\mathbf{c} H P L+A G$ group, $\mathbf{d} H P L+X G$ group (NB new bone, BM bone marrow, red arrow indicates boneto-implant contact, black arrow indicates residual xenograft particles) groups, RFA readings of implants were lower at baseline and were higher at 8 weeks. Also, no significant differences were found in RFA readings between AG and XG groups at 8 weeks (70.8 and 72.6, respectively) ( $p^{>} 0.05$ ). However, in HPL+AG group, RFA readings were lower than those of the HPL+XG group at 8 weeks $(p<0.05)$. RFA readings of HPL $+\mathrm{XG}$ group were not different compared to XG group $\left(p^{>} 0.05\right)$.

\section{Discussion}

Although bone regeneration is an efficient process in physiological conditions, many factors such as inflammation, hormonal changes, and also, elevated serum lipid levels associated with the impaired or delayed healing process. In the present study, an initial objective of the research was to identify the effects of hyperlipidemia on bone graft regeneration around peri-implant defects in rabbits. To the best of our knowledge, this is the first study to verify the inhibition of bone graft regeneration and implant stability in peri-implantal defect under hyperlipidemic conditions. And also, we demonstrated that the amount of bone-to-implant contact and implant stability improved in HPL+XG group compared to HPL + AG group.

A wound-healing situation that is unaffected by external factors such as microbial colonization, plaque accumulation is a prerequisite for evaluating potential regenerative or augmentation treatment modalities. Therefore, in our study, the tibia of a rabbit was selected for extraoral defect model. Rabbits are commonly used for screening implant materials prior to testing in a larger animal model because they are easy to handle, and due to the size of their tibia, allowing the creation of a large peri-implant defect and the installation of conventional implants in the proximal region of the tibia [15].

In literature, different experimental protocols have been used to induce hyperlipidemia and no available 


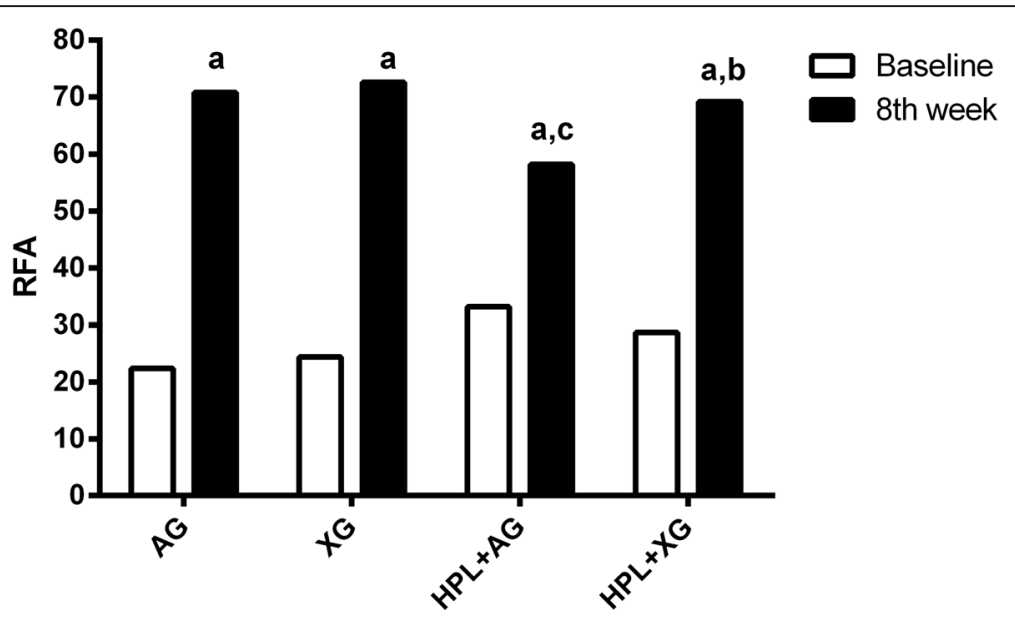

Fig. 3 Mean and standard deviation of RFA readings in study groups at baseline and 8 weeks. ${ }^{a} p<0.05$ versus baseline, ${ }^{b} p<0.05$ versus HPL + AG group, ${ }^{c} p<0.05$ versus AG group ( $n$ of each group is 5 )

threshold to confirm hyperlipidemia such as diabetic models that blood glucose levels were greater than 300 $\mathrm{mg} / \mathrm{dl}$. Earlier studies were used to induce hyperlipidemia by a different concentration or times such as a $1 \%$ cholesterol diet for 4 weeks [16], 1.5\% cholesterol diet for 12 weeks [17], and $0.5 \%$ cholesterol diet for 12 weeks [18]. Also, in a recent study [19], rabbits were fed a $2 \%$ cholesterol diet for 8 weeks, as we used in our study. After 8-week feeding, we observed a fivefold increase in total cholesterol and triglyceride levels in rabbits. Furthermore, these levels lasted during the study period as shown in Table 2.

Graft resorption called biodegradation should take place at a time appropriate to the activation of new bone formation [20]. Some of the studies showed that the xenograft was slowly resorbed or was encapsulated [20-22]. In another study that was investigated histologically the simultaneous placement of endosseous implants into the sinus cavity and the surgical elevation of the sinus floor including filling the cavity with different grafting materials, they found that hydroxyapatite or natural bovine bone mineral demonstrated newly formed bone with direct contact at the implant surface [12]. Similarly, our results showed that xenograft particles were almost replaced by new bone. But, bone-to-implant contact was decreased in the hyperlipidemic group. However, there is no clinical data available in which a clinical outcome obtained following regenerative therapy of hyperlipidemic patient appears to be more unfavorable than those of the non-hyperlipidemic patients. Only one retrospective study suggested that high total serum cholesterol levels tend to increase graft failure rates whilst it did not influence implant failures. In that study, only autologous and/ or deproteinized bovine bone was used for all cases, as we used in this study [2].

Hyperlipidemia increases the risk for the generation of lipid oxidation products. Pirih's animal study found that hyperlipidemia induces secondary hyperparathyroidism and impaired bone regeneration and mechanical strength through actions of oxidized lipids which accumulate in the subendothelial spaces of vasculature and bone [23]. Also, in a recent study, the authors evaluated the effects of hyperlipidemia on implant osseointegration in mice via micro-CT analysis [1]. They reported that HF diet-fed mice had significantly decreased bone formation and bone-to-implant contact. Interestingly, in our study, the HPL+AG group showed less BIC compared to the HPL+XG group, probably by upregulating osteoclastogenesis and suppressing mineralization. But keeping in mind that small sample size may affect these results and therefore it must be confirmed in future studies using a larger sample size.

Osseointegration and bone healing are similar processes as they both involve similar cells, hormones, and systems. Therefore, drugs or systemic diseases that affect bone healing can also decrease osseointegration [24]. In addition, the quality and quantity of the bone surrounding the implants are a critical factor to the long-term prognosis. There are several methods that have been purposed to evaluate implant osseointegration, such as the cutting torque resistance analysis and the resonance frequency analysis [25]. RFA analysis aims at providing an objective and non-invasive measurement, and also the RFA has been extensively used in experimental and clinical research [26]. However, Balshi et al. reported that RFA measurements were associated with the bone density, the location of placement, and the gender [27]. Furthermore, in a study that investigated the correlations between the RFA analysis and peri-implant bone levels in surgically created dehiscence defects and circumferential defects, they found that RFA readings were correlated to circumferential and wide-dehiscence defects but not for narrow-dehiscence defects. According 
to the authors, the size of surrounding bone defects was highly associated with RFA readings. Huang et al. suggested that the highest resonance frequency value was found when the implant was placed into the type I surrounding bone and in contrast, the resonance frequency of the implant with type IV bone quality was found almost fourfold less than that found in the type I model [28]. In our study, baseline RFA readings were decreased in all groups due to circumferential intra-bony defects (class 1-e, according to defect classifications of Schwarz et al.) and the wide bone marrow of the tibial bone and no significant differences among the groups [29].

In reviewing the literature, only one study has explored the effect of hyperlipidemia on implant stability in mice [1]. In that study, the most important finding was that high-fat diet-fed mice had increased implant loss and the high-fat diet group required a lower load to break the bone-to-implant contact at 4 and 8 weeks according to push-in test results. Also, in that study, there was no peri-implantal defect and all implants were inserted pristine bone of mice. However, our results showed that RFA readings were decreased in HPL+AG groups compared to controls, and RFA readings in the HPL+XG group were better results than that in the HPL + AG group. These results must be confirmed in a larger sample size because of a low sample size is the main limitation of this study.

In conclusion, within the limitations of this animal study, these findings support the hypothesis that hyperlipidemia impaired bone graft regeneration especially, autogenous graft healing with peri-implant defects and also decreased implant stability. Thus, according to our results, we confirmed the adverse effects of hyperlipidemia on implant osseointegration and peri-implant defect regeneration. However, future studies needed to investigate the mechanism of action of hyperlipidemia on graft materials in selecting appropriate graft materials for hyperlipidemic patients.

\section{Acknowledgements}

Not applicable

\section{Funding}

This study was supported by the Cumhuriyet University Unit of Scientific Research Projects (project number DIS-171).

\section{Availability of data and materials}

Not applicable

\section{Authors' contributions}

MBT performed the experimental protocol and histomorphometric analysis and was a major contributor in writing the manuscript. $\mathrm{HT}$ analyzed and interpreted the data. Both authors read and approved the final manuscript.

\section{Ethics approval and consent to participate}

This protocol was approved by the Animal Ethics Committee of Cumhuriyet University Faculty of Medicine under protocol number 65202830/130.
Consent for publication

Not applicable

Competing interests

Mehmet Bugrul Tekin and Hulya Toker declare that they have no competing interests.

\section{Publisher's Note}

Springer Nature remains neutral with regard to jurisdictional claims in published maps and institutional affiliations.

\section{Author details}

${ }^{1}$ Oral Health Center, Kayseri, Turkey. ${ }^{2}$ Periodontology Department, Gulhane Faculty of Dentistry, University of Health Sciences, Ankara, Turkey.

Received: 10 December 2018 Accepted: 15 March 2019

Published online: 15 May 2019

\section{References}

1. Keuroghlian A, Barroso ADV, Kirikian G, Bezouglaia O, Tintut Y, Tetradis S, et al. The effects of hyperlipidemia in implant osseointegration. J Oral Implantol. 2015;41(2):e7-e11.

2. Tirone F, Salzano S, D'Orsi L, Paola P, Rodi D. Is a high level of total cholesterol a risk factor for dental implants or bone grafting failure? A retrospective cohort study on 227 patients. Eur J Oral Implantol. 2016;9(1):77-84.

3. Saxlin T, Suominen-Taipale L, Kattainen A, Marniemi J, Knuuttila M, Ylöstalo P. Association between serum lipid levels and periodontal infection. J Clin Periodontol. 2008;35(12):1040-7.

4. Bilezikian JP, Brandi ML, Rubin M, Silverberg SJ. Primary hyperparathyroidism: new concepts in clinical, densitometric and biochemical features. J Intern Med. 2005;257(1):6-17.

5. Gaviria L, Salcido JP, Guda T, Ong JL. Current trends in dental implants. J Korean Assoc Oral and Maxillofac Surg. 2014;40(2):50-60.

6. Al-Subaie AE, Laurenti M, Abdallah MN, Tamimi I, Yaghoubi F, Eimar H, et al. Propranolol enhances bone healing and implant osseointegration in rats tibiae. J Clin Periodontol. 2016;43(12):1160-70.

7. Moriyama Y, Ayukawa Y, Ogino Y, Atsuta I, Todo M, Takao Y, Koyano K. Local application of fluvastatin improves peri-implant bone quantity and mechanical properties: a rodent study. Acta Biomater. 2010;6(4):1610-8.

8. García-Gareta E, Coathup MJ, Blunn GW. Osteoinduction of bone grafting materials for bone repair and regeneration. Bone. 2015;81:112-21.

9. Sanz-Sánchez I, Ortiz-Vigón A, Sanz-Martín I, Figuero E, Sanz M. Effectiveness of lateral bone augmentation on the alveolar crest dimension: a systematic review and meta-analysis. J Dental Res. 2015;94(9_suppl):128S-42S.

10. Cardaropoli D, Gaveglio L, Cardaropoli G. Vertical ridge augmentation with a collagen membrane, bovine bone mineral and fibrin sealer: clinical and histologic findings. Int J Periodont \& Restor Dent. 2013;33(5):583-9.

11. Berglundh T, Lindhe J. Healing around implants placed in bone defects treated with Bio-Oss ${ }^{\circledast}$. An experimental study in the dog. Clin Oral Implants Res. 1997:8(2):117-24.

12. Wetzel $A C$, Stich $H$, Caffesse RG. Bone apposition onto oral implants in the sinus area filled with different grafting materials. A histological study in beagle dogs. Clin Oral Implants Res. 1995:6(3):155-63.

13. Hockers T, Abensur D, Valentini P, Legrand R, Hämmerle CHF. The combined use of bioresorbable membranes and xenografts or autografts in the treatment of bone defects around implants. A study in beagle dogs. Clin Oral Implants Res. 1999:10(6):487-98.

14. RD\&l Christchurch. Pekapeka / bats. Te Papa Atawhai: Department of Conservation; 2005

15. dos Santos PL, de Molon RS, Queiroz TP, Okamoto R, de Souza Faloni AP, Gulinelli $J$, et al. Evaluation of bone substitutes for treatment of periimplant bone defects: biomechanical, histological, and immunohistochemical analyses in the rabbit tibia. J Periodontal \& Implant Sci. 2016;46(3):176-96

16. Drew AF, Tipping PG. Cyclosporine treatment reduces early atherosclerosis in the cholesterol-fed rabbit. Atherosclerosis. 1995;116(2):181-9.

17. Matsumoto T, Saito E, Watanabe H, Fujioka T, Yamada T, Takahashi Y, et al Influence of FK506 on experimental atherosclerosis in cholesterol-fed rabbits. Atherosclerosis. 1998;139(1):95-106. 
18. Roselaar SE, Schonfeld G, Daugherty A. Enhanced development of atherosclerosis in cholesterol-fed rabbits by suppression of cell-mediated immunity. J Clin Invest. 1995;96(3):1389.

19. Chen Y-H, Lin W-W, Liu C-S, Hsu L-S, Lin Y-M, Su S-L. Caveolin-1 expression ameliorates nephrotic damage in a rabbit model of cholesterol-induced hypercholesterolemia. PLoS One. 2016;11(4):e0154210.

20. Yildirim M, Spiekermann H, Biesterfeld S, Edelhoff D. Maxillary sinus augmentation using xenogenic bone substitute material Bio-Oss in combination with venous blood. A histologic and histomorphometric study in humans. Clin Oral Implants Res. 2000;11(3):217-29.

21. Poulias E, Greenwell H, Hill M, Morton D, Vidal R, Shumway B, Peterson TL. Ridge preservation comparing socket allograft alone to socket allograft plus facial overlay xenograft: a clinical and histologic study in humans. J Periodontol. 2013;84(11):1567-75.

22. Skoglund A, Hising P, Young C. A clinical and histologic examination in humans of the osseous response to implanted natural bone mineral. Int J Oral Maxillofac Implants. 1997;12(2):194-9.

23. Pirih F, Lu J, Ye F, Bezouglaia O, Atti E, Ascenzi MG, et al. Adverse effects of hyperlipidemia on bone regeneration and strength. J Bone and Mineral Res. 2012:27(2):309-18.

24. Feller $L$, Chandran R, Khammissa RAG, Meyerov R, Jadwat $Y$, Bouckaert $M$, et al. Osseointegration: biological events in relation to characteristics of the implant surface: clinical review. South African Dental J. 2014;69(3):112-7.

25. Östman P-O, Hellman M, Wendelhag I, Sennerby L, et al. Resonance frequency analysis measurements of implants at placement surgery. Int $J$ Prosthodont. 2006;19(1):77-83.

26. Chan H-L, Misch K, Wang H-L. Dental imaging in implant treatment planning. Implant Dent. 2010;19(4):288-98

27. Balshi SF, Allen FD, Wolfinger GJ, Balshi TJ. A resonance frequency analysis assessment of maxillary and mandibular immediately loaded implants. Int J Oral Maxillofac Surg. 2005;20(4):584-94.

28. Huang HM, Lee SY, Yeh CY, Lin CT. Resonance frequency assessment of dental implant stability with various bone qualities: a numerical approach Clin Oral Implants Res. 2002;13(1):65-74

29. Schwarz F, Herten M, Sager M, Bieling K, Sculean A, Becker J. Comparison of naturally occurring and ligature-induced peri-implantitis bone defects in humans and dogs. Clin Oral Implants Res. 2007;18(2):161-70.

\section{Submit your manuscript to a SpringerOpen ${ }^{\circ}$ journal and benefit from:}

- Convenient online submission

- Rigorous peer review

- Open access: articles freely available online

- High visibility within the field

- Retaining the copyright to your article

Submit your next manuscript at $\boldsymbol{\nabla}$ springeropen.com 